Volume 9, No.1, January - February 2020

International Journal of Advanced Trends in Computer Science and Engineering

Available Online at http://www.warse.org/IJATCSE/static/pdf/file/ijatcse109912020.pdf

https://doi.org/10.30534/ijatcse/2020/109912020

\title{
Algorithmic Analytics for Outcomes-based Tertiary Education Performance Assessment
}

\author{
Glendell R. Jadraque $^{1^{*}}$, Allemar Jhone P. Delima ${ }^{2}$, Ramcis N. Vilchez ${ }^{3}$ \\ ${ }^{1,2}$ Professional Schools, University of Mindanao, Matina, Davao City, Davao del Sur, Philippines \\ ${ }^{3}$ College of Computing Education, University of Mindanao. Matina, Davao City, Davao del Sur, Philippines \\ 'gjadraque@umindanao.edu.ph, ${ }^{2}$ allemardelima@umindanao.edu.ph, ${ }^{3}$ ramcis_vilchez@umindanao.edu.ph
}

\begin{abstract}
Global educational institutions are continuously working to enhance the needed curricula to answer the demands of state and business enterprise. They are now working with Outcomes-based Education (OBE), which denies the traditional way of education to students. Instead, it makes students show that they are capable of doing the required learning outcomes. The quest for learning the correct assessment for education while under the era of OBE is still an on-going call. With the help of Educational Data Mining, a real-world dataset of 549 were trained from the student record. The study intends to identify the probability of the student to pass practical evaluation following OBE methodology, using selected WEKA-based classifiers, namely Naive Bayes, C4.5, and Random Forest. The said classifiers revealed promising accuracy of $78.10 \%$ for Naïve Bayes, $93.07 \%$ for C4.5, and $95.99 \%$ for Random Forest. Hence, the EDM's future is really for further study, which can be implemented in the fields of entertainment, industry, medicine, and many others because of massive raw databases.
\end{abstract}

Key words: C4.5, EDM, Naïve Bayesian, Outcomes-based education, Random Forest

\section{INTRODUCTION}

Outcomes-based Education is a student-focused, results-based way of dealing with learning [1]. It steers evaluation towards specific targets, namely what students ought to take care of and what merits understanding in a content-heavy educational program [2]. Educators and students center on the ultimate ideal outcomes of each learning procedure, which continuously evaluates the discovery of whether they are gaining any grounds or not [1].

An essential concern to be asked by an OBE program product to instruction and education is if they are capable of using what they have studied in a realistic environment, which attempts to explore higher-order thinking skills competencies and their meanings used in OBE [1].

With the intense focus on desired education outcomes in higher education [3], academic institutions all over the world have been under growing strain from governments to exhibit competence, furthermore, cost-effectiveness through more significant and precise reporting of program outcomes [4]. In nations, for example, Australia [2], [3], Canada [5], the United States [3], [5], the UK [3], South Africa [1], and New Zealand [2], [3], [5], OBE is, at present, implemented and supported globally to elevate educational renovation [5]. The emphasis considering learning outcomes has given a lift to interests about the extreme weight on the conducive and economic worth of education that is measured through competence and effectiveness in the contemporary educational context [3].

Learning assessment is, therefore, the essential methodology by which the desired learning outcomes described by a unit of learning, indeed credited as the discovering significance [6] and the authentic end-results or worth earned by a learner, that should mold from potential to actual [7]. It is promoted by research that the means of assessment should be for quality before deployment [1]. There are varied yet relevant kinds of evaluation in academic institutions, including assessment, end-results, attrition analysis, and retention. Also, there are some refined alternative models of evaluation, including curriculum-based [8], outcomes-based [9], [10], and performance-based assessment [11], [6].

Concerning education today, the utilization of Data Mining is fit [12] for data learning, decisions-support, and instruction [13]. The application of DM in the field of education is developing. It serves as the beginning of educational data mining (EDM) study [14] because instead of seeking natural resources, it targets educational knowledge [13], [15] to adequately know the student learning setting [16]. EDM does various data mining methods such as Neural Networks (NN) [15], Decision Trees (DT), Naïve Bayes (NB) [17], K- Nearest Neighbor (KNN), Random Forest (RF), Support Vector Machine (SVM) [17], and many others [15], [18], [19].

The study concerns the prediction analysis of student's performance assessment under the rig of Outcomes-based Education (OBE). In determining the possible outcomes systematically, the study used Naïve Bayes [17], C4.5, and Random Forest [16]. Through this, it will generate an imminent approach as to how likely a student will right-fit to OBE through data interpretation and student performance, given the implementation of OBE methodology.

Figure 1 shows several significant measures included in OBE, including determining assessment measures. 


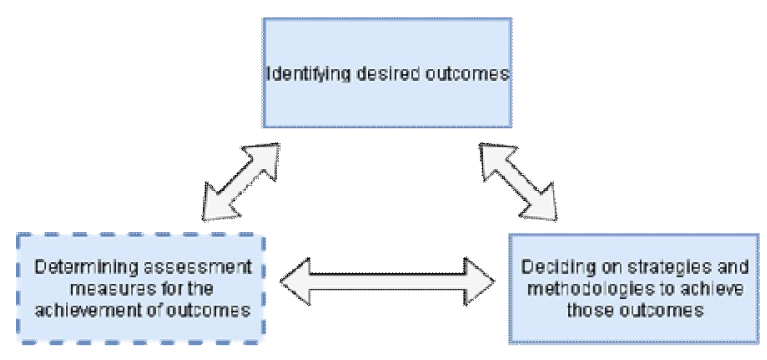

Figure 1: OBE being an Approach [17]

\section{LITERATURE REVIEW}

\subsection{Review Stage}

In working this research out, a literature review holds out to interpret its current position. This is to support the idea gathered in redefining the contextual basis for OBE assessment data analysis.

Educational Data Mining (EDM) is still new in terms of Data Mining procedures [16], which has been introduced as a future research field correlated to various buttoned-down operations of studies, including AH, DM, e-learning, ITSs, WM, and many others [20]. EDM can also work in areas such as accounts, politics, sports, transportation [16], business, genetics, medicine [20], and a lot more [16], [20] because it merely reflects exact ways as the overall DM process such as preprocessing, DM, and postprocessing [20]. As stated above, EDM uses various data mining techniques. Hence, it helps discover knowledge from data coming from the educational environment [21].

\subsection{Classifiers}

\section{A. Nä̈ve Bayes}

As described by the author in [18], that, since, Naïve Bayes (NB) is the most famous analysis algorithm, due to its plainness [22] and efficiency that matches the concept of probabilities, it is applied in labeling data for analysis purposes [18]. There was a classification as positive or negative based on their ratings using movie reviews. Following the experimental evaluations, the system scored an accuracy of $83 \%$. Another application of NB used a training set consists of 1500 questions for every 20 classes of the newsgroup. NB achieved an accuracy of 1 for class 1 and 0.58 for class 2 . The same training set used, and SVM generated a different accuracy of 0.95 for both classes 1 and 2 [17]. The study commands a different method in getting the probability of the instance given with a precise dataset [22]. As it said on [23], Naïve Bayes is frequently applied as a baseline classifier, which measures other classifiers that consistently gives rational classification performance [23].

As shown in equation (1), a dependent probability is a probability that case " $\mathrm{c}$ " will happen, given the proof " $\mathrm{x}$ ", which written usually as $\mathrm{P}(\mathrm{c} \mid \mathrm{x})$. The Naïve Bayes Theorem permits to define the odds when all left is the probability of the contrary conclusion and the two elements only [24]:

$$
P(c \mid x)=\frac{P(x \mid c) P(c)}{P(x)}
$$

When attempting to determine the probability of things, this restatement (1) can stay pretty significant based on occurring instances [24].

\section{B. $C 4.5$}

Algorithms [16], [25], including C4.5 or J48 in WEKA, were used in classifying similarly [26], predicting attainment [25], and achieving returns from hypothesis experimentation [16]. It showed that the type of schools does not affect student performance, but it is the parent's job who plays a significant part in predicting grades [16]. A study for university students revealed that the C4.5 decision tree algorithm is used in the prediction, analysis, and prevention of their academic failure, specifically, examination failure [20].

Retrieved results from the implementation of the $\mathrm{C} 4.5$ algorithm in the university containing students' records [26], as shown in Figure 2.

\begin{tabular}{|lcc|}
\hline == Surmary $===$ & & \\
& & $87.7551 \%$ \\
Correct1y Classified Instances & 43 & $12.2449 \%$ \\
Incorrect1y Classified Instances & 6 & \\
Kappa statistic & 0.7977 & \\
Mean absolute error & 0.0939 & \\
Root mean squared error & 0.2167 & \\
Relative absolute error & $29.9787 \&$ \\
Root relative squared error & $55.1647 \&$ & \\
Coverage of cases (0.95 level) & $97.9592 \&$ \\
Mean rel. region size (0.95 leve1) & $39.2857 \&$ \\
Total Number of Instances & 49 & \\
\end{tabular}

Figure 2: Generated Results after the Implementation of C4.5 Decision Tree [26]

Compared to other algorithms [27] like the k-means clustering technique [26], C4.5 is a single custom of foretelling end-results that promptly creates decision trees with high precision, yet it is more when it comes to cost-effectiveness [27] and efficiency [26].

Comparison of various classification techniques. Both C4.5 and Naïve Bayes went on top [17], as shown in Table 1.

Table 1. Comparative Interpretation of Classification Techniques [17]

\begin{tabular}{cccccc}
\hline Classifier & $\begin{array}{c}\text { Processing Time } \\
\text { (sec) }\end{array}$ & $\begin{array}{c}\text { Correctly } \\
\text { classified } \\
\text { instances (\%) }\end{array}$ & $\begin{array}{c}\text { Incorrectly } \\
\text { classified } \\
\text { instances (\%) }\end{array}$ & $\begin{array}{c}\text { Kappa statistic } \\
\text { Root mean } \\
\text { square error }\end{array}$ \\
\hline J48 & 0.02 & 90 & 10 & 0.7692 & 0.146 \\
error \\
Naïve Bayes & 0.01 & 85 & 15 & 0.625 & 0.3018 \\
OneR & 0 & 70 & 30 & 0.3496 & 0.3 \\
ZeroR & 0 & 70 & 31.6667 & 0.2339 & 0.4238 \\
Ibk & 0 & 68.3333 & 0.4594 & 0.3312 \\
\hline
\end{tabular}




\section{Random Forest}

One of the fittest algorithms is Decision Trees, in terms of data classification, giving high accuracy for various problems in a comparatively brief time [28]. Both DTs and Naïve Bayes are used in EDM [16]. DT termed as decision support media that are typically done in decision study problems to support the classification of the most right-fit approach for attaining a solid goal [28].

[18] also mentioned the importance of Random Forest and matched its review with other classifiers. [18] supports the claims of [29] that the Random Forest algorithm gives practical and discriminative analysis resulting in a point that it is considered a competent classifier. RF was used to predict ultimate student production and forecast, which students might not pass [13] or as termed by [20], "drop out". In terms of micro average, RF is also known for its optimal performance [18].

Just like Naïve Bayes and C4.5, Random Forest has been used to foretell the students' outcomes based on a suggested guide, as mentioned in [30].

Data set evaluation results using C4.5, Naïve Bayes, and Random Forest Algorithms [30], as shown in Table 2.

Table 2. Results after the Evaluation of Dataset used in [30]

\begin{tabular}{|c|c|c|c|c|c|c|c|c|}
\hline 䍘 & 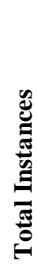 & 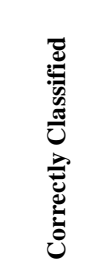 & 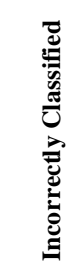 & 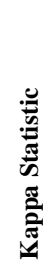 & 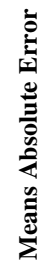 & 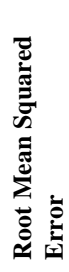 & 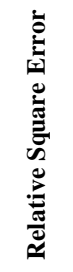 & 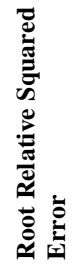 \\
\hline $\mathrm{J} 48$ & $\begin{array}{l}6 \\
0\end{array}$ & $\begin{array}{l}56(93.3 \\
333 \%)\end{array}$ & $\begin{array}{l}4(6.66 \\
67 \%)\end{array}$ & $\begin{array}{r}0.8 \\
821\end{array}$ & $\begin{array}{r}0.0 \\
81\end{array}$ & $\begin{array}{r}0.2 \\
012\end{array}$ & $\begin{array}{r}20.79 \\
86 \%\end{array}$ & $\begin{array}{r}45.75 \\
77 \%\end{array}$ \\
\hline $\begin{array}{l}\text { Naïv } \\
\text { e }\end{array}$ & 6 & $\begin{array}{l}52(86.6 \\
667 \%)\end{array}$ & $8(13.3$ & $\begin{array}{r}0.7 \\
688\end{array}$ & $\begin{array}{r}0.1 \\
16\end{array}$ & $\begin{array}{r}0.2 \\
369\end{array}$ & $\begin{array}{r}29.79 \\
28 \%\end{array}$ & $\begin{array}{r}53.87 \\
98 \%\end{array}$ \\
\hline $\begin{array}{l}\mathrm{s} \\
\text { Rand }\end{array}$ & & & & & & & & \\
\hline $\begin{array}{l}\text { om } \\
\text { Fore } \\
\text { st } \\
\text { tree }\end{array}$ & $\begin{array}{l}6 \\
0\end{array}$ & $\begin{array}{l}60(100 \\
\%)\end{array}$ & $0(0 \%)$ & 1 & $\begin{array}{r}0.0 \\
804\end{array}$ & $\begin{array}{r}0.1 \\
175\end{array}$ & $\begin{array}{r}20.65 \\
76 \%\end{array}$ & $\begin{array}{r}26.71 \\
67 \%\end{array}$ \\
\hline
\end{tabular}

\section{METHODOLOGY}

\subsection{Process}

When it comes to giving importance to students' scores, this study is anchored to [31], [32] about the type of assessment system being used in outcomes-based [31]. This is also supported by [33], especially on higher education [34], which is preparatory for each individual to the real-working world [14], [33].

Once the current EDM progress improvement, it is conserved and developed. Then, it will undergo content-organization, content-analyzation, and content-discussion of the study based on end-results provided by a DM approach [13]. This study used the application of the Knowledge Discovery Process, which was also performed by [15], [16], [18], [30], [26], as shown in Figure 3.

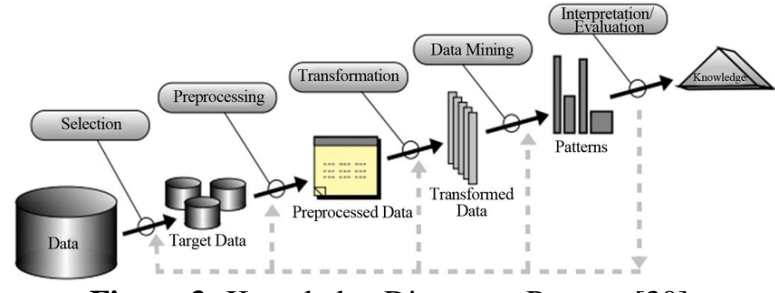

Figure 3: Knowledge Discovery Process [30]
Using the type of data-to-information transformation approach mentioned above [15], [30]. This gathered the records of first-year, Bachelor of Science in Information Systems and Information Technology students of Davao del Norte State College, for the academic years 2018-2019 and 2019-2020. The data gathered were used for prediction analysis, as shown in Figure 4, which is anchored to the conceptual framework solely dedicated to this study.

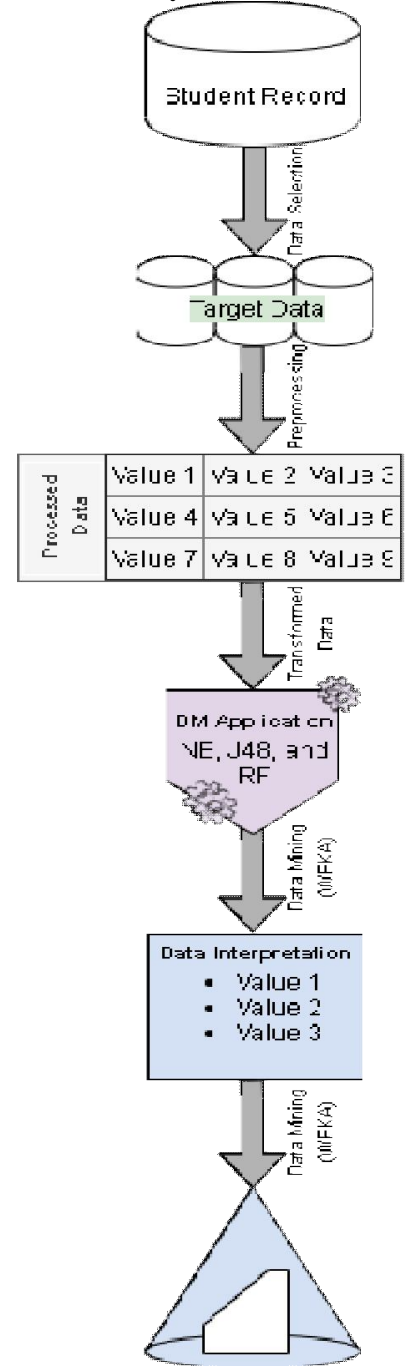

Figure 4: Conceptual Structure of the Study

\subsection{Datasets}

In this study, a sum of 549 students' records from the two sets of 1st-year class records of BSIS and BSIT, at DNSC, 1st semester, academic years from 2018-2020, were used as datasets which are shown in Table 3. The data obtained were cross-referenced from the Institute of Information Technology records held by the Program Chairpersons and database of the Integrated Academic Information Management System (IAIMS) that will be trained to achieve optimal accuracy for data mining.

Table 3. Data specifics

\begin{tabular}{ccccccccc}
\hline \multirow{2}{*}{ AY } & \multicolumn{4}{c}{ BSIT } & \multicolumn{4}{c}{ BSIS } \\
& Set & Set & Set & Set & Set & Set & Set & Total \\
& A & B & C & D & E & A & B & \\
\hline $2018-2019$ & 40 & 40 & 40 & 39 & 38 & 41 & 40 & 278 \\
$2019-2020$ & 40 & 39 & 38 & 38 & 35 & 41 & 40 & 271 \\
\hline
\end{tabular}




\subsection{Data Preprocessing}

To add more precision, data preprocessing was performed by challenging the variables, as shown in Table 4 and their data [14][21][27]. This is to make the mining method less confused when it comes to the identification, interpretation, and analysis of data. As per the academic year: 2018-2019 enrollment report, there is a total of 278 students, and the academic year: 2019-2020 enrollment has 271 after official and unofficial dropouts.

Table 4. Assessment-related variables with description and possible values

\begin{tabular}{|c|c|c|c|}
\hline Variable & Description & Possible Value & $\begin{array}{l}\text { Class } \\
\text { Type }\end{array}$ \\
\hline Age & Student's age & $\begin{array}{c}\mathrm{a}=17-19 \text { yrs. old; } \\
\mathrm{b}=20-21 \text { yrs. old; } \\
\& \mathrm{c}=22 \text { yrs. old } \\
\text { and up }\end{array}$ & $\begin{array}{c}\text { Multi-cla } \\
\text { ss }\end{array}$ \\
\hline Gender & Student's gender & Male; Female & Binary \\
\hline Type of Learner & $\begin{array}{l}\text { The type of } \\
\text { learner the } \\
\text { student is }\end{array}$ & $\begin{array}{c}\text { Auditory; } \\
\text { Kinesthetic; } \\
\text { Visual }\end{array}$ & $\begin{array}{l}\text { Multi-cla } \\
\text { ss }\end{array}$ \\
\hline K-12 Grad & $\begin{array}{l}\text { If the student } \\
\text { went in the old } \\
\text { or new } \\
\text { curriculum }\end{array}$ & Yes; No & Binary \\
\hline SHS Strand (ICT) & $\begin{array}{l}\text { If graduate of } \\
\text { SHS, does the } \\
\text { student have the } \\
\text { strand of ICT }\end{array}$ & Yes; No & Binary \\
\hline $\begin{array}{c}\text { Resource } \\
\text { Availability }\end{array}$ & $\begin{array}{c}\text { DNSC's } \\
\text { computer } \\
\text { laboratory to } \\
\text { students ratio \% }\end{array}$ & $\begin{array}{c}\%(\text { Headcount } / \text { To } \\
\text { tal \# of available } \\
\text { computer units) } \\
a=70 \%-80 \% ; \\
b=80.99 \%-90 \% \\
c=90.99 \%-100 \%\end{array}$ & $\begin{array}{c}\text { Multi-cla } \\
\text { ss }\end{array}$ \\
\hline $\begin{array}{c}\text { Owned Personal } \\
\text { Unit }\end{array}$ & $\begin{array}{l}\text { If the student has } \\
\text { his/her own PC }\end{array}$ & Yes; No & Binary \\
\hline Class Attendance & $\begin{array}{l}\text { Student's class } \\
\text { attendance }\end{array}$ & $\begin{array}{c}\%(\text { No. of } \\
\text { instance } \\
\text { (Present)/Total \# } \\
\text { of meetings) } \\
a=70 \%-80 \% ; \\
b=80.99 \%-90 \% ; \\
c=90.99 \%-100 \%\end{array}$ & $\begin{array}{l}\text { Multi-cla } \\
\text { ss }\end{array}$ \\
\hline $\begin{array}{c}\mathrm{Q}, \mathbf{A}, \mathbf{C P}, \boldsymbol{\&} \\
\operatorname{Pr}=100 \%\end{array}$ & $\begin{array}{l}\text { Average of } \\
\text { student's } \\
\text { quizzes, } \\
\text { assignment, } \\
\text { class } \\
\text { participation, }\end{array}$ & $\begin{array}{c}a=70 \%-80 \% \\
b=80.99 \%-90 \% \\
c=90.99 \%-100 \%\end{array}$ & $\begin{array}{c}\text { Multi-cla } \\
\text { ss }\end{array}$ \\
\hline
\end{tabular}

\begin{tabular}{|c|c|c|c|}
\hline & $\begin{array}{c}\text { and } \\
\text { project }=100 \%\end{array}$ & & \\
\hline $\begin{array}{c}\text { Paper \& Pen } \\
\text { Evaluation-0.4\% }=10 \\
0 \%\end{array}$ & $\begin{array}{c}\text { Student's } \\
\text { evaluation range } \\
\text { from the } \\
\text { traditional paper } \\
\& \text { pen } \\
\text { examination }=10 \\
0 \%\end{array}$ & $\begin{array}{c}a=70 \%-80 \% \\
b=80.99 \%-90 \% \\
c=90.99 \%-100 \%\end{array}$ & $\begin{array}{c}\text { Multi-cla } \\
\text { ss }\end{array}$ \\
\hline $\begin{array}{c}\text { Practicum } \\
\text { Evaluation-0.6\% }=10 \\
0 \%\end{array}$ & $\begin{array}{c}\text { Student's } \\
\text { evaluation range } \\
\text { from the } \\
\text { laboratory } \\
\text { examination } \\
\text { under } \\
\text { OBE }=100 \%\end{array}$ & $\begin{array}{c}\text { low }=70 \%-80 \% \\
\text { ave }=80.99 \%-90 \\
\% ; \\
\text { high }=90.99 \%-10 \\
0 \%\end{array}$ & $\begin{array}{c}\text { Multi-cla } \\
\text { ss }\end{array}$ \\
\hline
\end{tabular}

\subsection{Data Mining}

DM can give extensive yet specific prediction and decision-making, which is applicable in the field of academes, such as students' grades, GPA, drop rate, recommendation, and many others [12]. In achieving machine learning and DM, a modern tool applicable to education, WEKA toolkit has to be employed [16]. WEKA holds an extensive set of advanced Java-based ML and DM algorithms [14]. It includes instruments for and visualization. In making the gathered data compatible upon using the WEKA DM toolkit, it has to be prepared and changed to (.arff) file format [14][27].

\section{RESULT AND DISCUSSION}

With the application of C4.5, Naive Bayesian, and Random Forest algorithms through 5-fold and 10-fold Cross-Validation and Percentage Split of 70\%:30\% test options in WEKA, the training of datasets showed competitive results, which are somehow similar to the existing studies used, for example [30]. A sample of the dataset used for training is shown in Table 5 .

Table 5. Sample from the entire dataset

\begin{tabular}{|c|c|c|c|c|c|c|c|c|c|c|c|}
\hline $\begin{array}{c}\mathrm{Ag} \\
\mathrm{e}\end{array}$ & $\begin{array}{c}\text { Gend } \\
\text { er }\end{array}$ & $\begin{array}{l}\text { Type of } \\
\text { Learner }\end{array}$ & $\begin{array}{c}\text { K-1 } \\
2 \\
\text { Gra } \\
\text { d }\end{array}$ & $\begin{array}{l}\text { SHS } \\
\text { Stran } \\
\text { d } \\
(\text { ICT })\end{array}$ & $\begin{array}{l}\text { Resource } \\
\text { Availabili } \\
\text { ty } \%\end{array}$ & $\begin{array}{l}\text { Owned } \\
\text { Person } \\
\text { al Unit }\end{array}$ & $\begin{array}{c}\text { Cours } \\
\text { e } \\
\text { Subjec } \\
\text { t } \\
\text { Retak } \\
\text { er }\end{array}$ & $\begin{array}{l}\text { Class } \\
\text { Attendan } \\
\text { ce } \%\end{array}$ & $\begin{array}{c}\text { Q, A, } \\
\text { CP, \& } \\
\operatorname{Pr}=100 \\
\%\end{array}$ & $\begin{array}{c}\text { Paper \& Pen } \\
\text { Evaluation-0.4\% =1 } \\
00 \%\end{array}$ & $\begin{array}{c}\text { Practicum } \\
\text { Evaluation-0.6\% }=1 \\
00 \%\end{array}$ \\
\hline $\mathrm{a}$ & male & visual & yes & yes & $\mathrm{b}$ & no & no & $\mathrm{c}$ & $\mathrm{b}$ & $\mathrm{b}$ & high \\
\hline $\mathrm{a}$ & female & auditory & yes & no & b & no & no & $\mathrm{c}$ & b & $\mathrm{a}$ & high \\
\hline $\mathrm{a}$ & female & $\begin{array}{l}\text { kinesthet } \\
\text { ic }\end{array}$ & yes & yes & $\mathrm{b}$ & yes & no & $\mathrm{c}$ & $\mathrm{c}$ & $\mathrm{b}$ & high \\
\hline $\mathrm{a}$ & male & visual & yes & yes & b & no & no & $\mathrm{c}$ & $\mathrm{c}$ & b & high \\
\hline $\mathrm{a}$ & female & auditory & yes & no & $\mathrm{b}$ & no & no & $\mathrm{c}$ & b & $\mathrm{b}$ & high \\
\hline $\mathrm{b}$ & male & visual & yes & no & $\mathrm{b}$ & no & no & $\mathrm{c}$ & b & $\mathrm{a}$ & low \\
\hline$\cdots$ & $\cdots$ & $\cdots$ & $\cdots$ & $\cdots$ & $\cdots$ & $\cdots$ & $\cdots$ & $\cdots$ & $\cdots$ & $\cdots$ & $\cdots$ \\
\hline$\ldots$ & $\ldots$ & $\ldots$ & $\ldots$ & $\ldots$ & $\ldots$ & $\ldots$ & $\ldots$ & $\ldots$ & $\ldots$ & $\ldots$ & $\ldots$ \\
\hline
\end{tabular}


As shown in Figure 5, WEKA pictures the allocation of values of the student records.

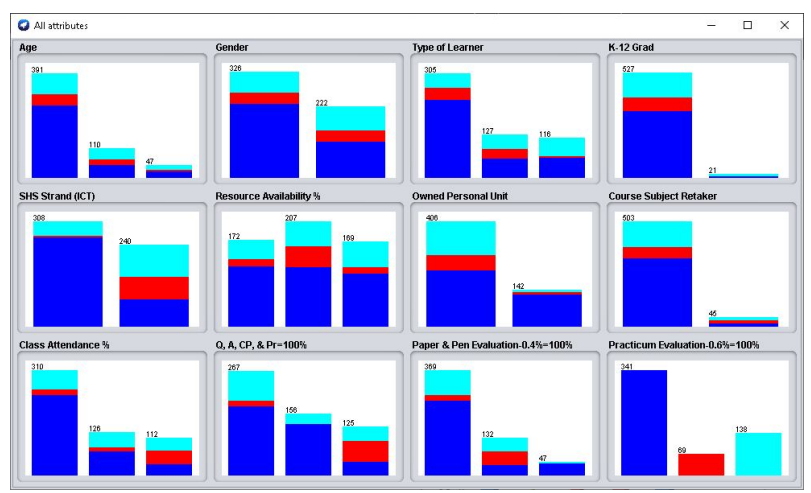

Figure 5: Students' Records Categorization Visualization

In predicting the student's performance, some tests were performed to assess the achievement and excellence of various classification algorithms [14]. Each algorithm has undergone each test option, as mentioned above, including Naïve Bayes that is shown in Table 6.

Table 6. Naïve Bayes' results specifics on applied test options

\begin{tabular}{|c|c|c|c|}
\hline Summary & $\begin{array}{c}\text { 5-fold } \\
\text { Cross-vali } \\
\text { dation }\end{array}$ & $\begin{array}{c}\text { 10-fold } \\
\text { Cross-vali } \\
\text { dation }\end{array}$ & $\begin{array}{c}\text { Percentage } \\
\text { Split } \\
(70 \%: 30 \%)\end{array}$ \\
\hline $\begin{array}{l}\text { Correctly Classified } \\
\text { Instances }\end{array}$ & $\begin{array}{c}426 \\
(77.7372 \%)\end{array}$ & $\begin{array}{c}428 \\
(78.1022 \%)\end{array}$ & $115(70.122 \%)$ \\
\hline $\begin{array}{l}\text { Incorrectly } \\
\text { Classified Instances }\end{array}$ & $\begin{array}{c}122 \\
(22.2628 \%)\end{array}$ & $\begin{array}{c}120 \\
(21.8978 \%)\end{array}$ & $49(29.878 \%)$ \\
\hline Kappa statistic & 0.5845 & 0.5937 & 0.4582 \\
\hline Mean absolute error & 0.1919 & 0.1916 & 0.2145 \\
\hline $\begin{array}{l}\text { Root mean squared } \\
\text { error }\end{array}$ & 0.3207 & 0.3202 & 0.3499 \\
\hline $\begin{array}{l}\text { Relative absolute } \\
\text { error }\end{array}$ & $53.8542 \%$ & $53.7944 \%$ & $59.7609 \%$ \\
\hline $\begin{array}{l}\text { Root relative } \\
\text { squared error }\end{array}$ & $76.0531 \%$ & $75.9208 \%$ & $82.0225 \%$ \\
\hline $\begin{array}{l}\text { Total Number of } \\
\text { Instances }\end{array}$ & \multicolumn{2}{|c|}{548} & 164 \\
\hline $\begin{array}{c}\text { Detailed } \\
\text { Accuracy By } \\
\text { Class (Weighted } \\
\text { Avg.) }\end{array}$ & $\begin{array}{c}\text { 5-fold } \\
\text { Cross-vali } \\
\text { dation }\end{array}$ & $\begin{array}{c}\text { 10-fold } \\
\text { Cross-vali } \\
\text { dation }\end{array}$ & $\begin{array}{c}\text { Percentage } \\
\text { Split } \\
(\mathbf{7 0 \%}: 30 \%)\end{array}$ \\
\hline TP Rate & 0.777 & 0.781 & 0.701 \\
\hline FP Rate & 0.16 & 0.159 & 0.223 \\
\hline Precision & 0.782 & 0.787 & 0.705 \\
\hline Recall & 0.777 & 0.781 & 0.701 \\
\hline F-Measure & 0.779 & 0.784 & 0.703 \\
\hline MCC & 0.616 & 0.617 & 0.472 \\
\hline ROC Area & 0.89 & 0.887 & 0.845 \\
\hline PRC Area & 0.841 & 0.836 & 0.810 \\
\hline Class & & high/low/a & \\
\hline
\end{tabular}

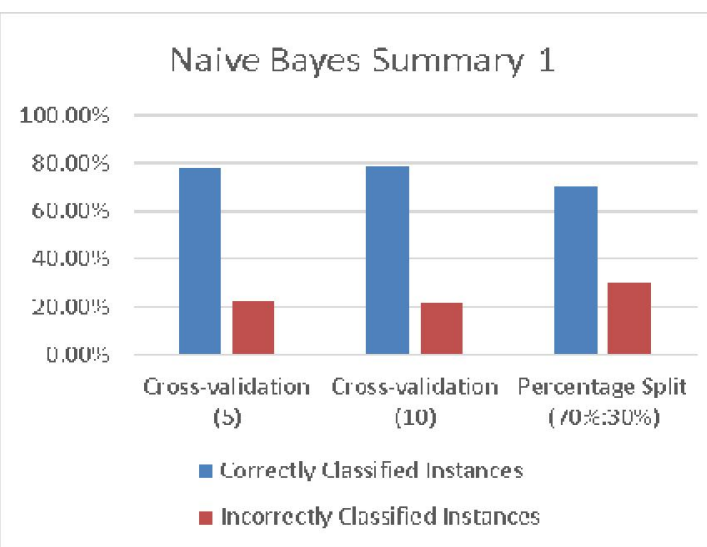

Figure 6: Naïve Bayes Test Options Results Visual Representation

Amongst the three-Test Options used in dataset training, the 10-fold Cross-validation that gave a promising result of $78.1 \%$ for Correctly Classified Instance, as shown in Figure 6.

A set of analyses were performed on the same Test Options employing $\mathrm{C} 4.5$ and RF algorithms, which presented assuring results. A tabular and graphical representation of results of both C4.5 and RF classifiers after 5 and 10-fold CV and PS of 70\%:30\% are shown in Figures 7 and 8 and Tables 7 and 8.

Table 7. C4.5's results specifics on applied test options

\begin{tabular}{|c|c|c|c|}
\hline Summary & $\begin{array}{c}\text { 5-fold } \\
\text { Cross-vali } \\
\text { dation }\end{array}$ & $\begin{array}{c}\text { 10-fold } \\
\text { Cross-vali } \\
\text { dation }\end{array}$ & $\begin{array}{c}\text { Percentage } \\
\text { Split } \\
(\mathbf{7 0 \%} \% \mathbf{3 0 \%})\end{array}$ \\
\hline $\begin{array}{l}\text { Correctly Classified } \\
\text { Instances }\end{array}$ & $\begin{array}{c}504 \\
(91.9708 \%)\end{array}$ & $\begin{array}{c}510 \\
(93.0657 \%)\end{array}$ & $137(83.5366 \%)$ \\
\hline $\begin{array}{l}\text { Incorrectly } \\
\text { Classified Instances }\end{array}$ & $\begin{array}{c}44 \\
(8.0292 \%)\end{array}$ & $38(6.9343 \%)$ & $27(16.4634 \%)$ \\
\hline Kappa statistic & 0.8466 & 0.8682 & 0.6921 \\
\hline Mean absolute error & 0.0527 & 0.433 & 0.1168 \\
\hline $\begin{array}{l}\text { Root mean squared } \\
\text { error }\end{array}$ & 0.1893 & 0.1681 & 0.2996 \\
\hline $\begin{array}{l}\text { Relative absolute } \\
\text { error }\end{array}$ & $14.7866 \%$ & $12.1625 \%$ & $32.5388 \%$ \\
\hline $\begin{array}{l}\text { Root relative } \\
\text { squared error }\end{array}$ & $44.899 \%$ & $39.8589 \%$ & $70.2220 \%$ \\
\hline $\begin{array}{l}\text { Total Number of } \\
\text { Instances }\end{array}$ & \multicolumn{2}{|c|}{548} & 164 \\
\hline
\end{tabular}

\begin{tabular}{lccc}
$\begin{array}{c}\text { Detailed } \\
\text { Accuracy By } \\
\text { Class (Weighted } \\
\quad \text { Avg.) }\end{array}$ & $\begin{array}{c}\text { 5-fold } \\
\text { Cross-vali } \\
\text { dation }\end{array}$ & $\begin{array}{c}\text { 10-fold } \\
\text { Cross-vali } \\
\text { dation }\end{array}$ & $\begin{array}{c}\text { Percentage } \\
\text { Split } \\
(\mathbf{7 0 \%} \% \mathbf{3 0 \% )}\end{array}$ \\
TP Rate & 0.92 & 0.931 & 0.835 \\
FP Rate & 0.07 & 0.061 & 0.138 \\
Precision & 0.918 & 0.930 & 0.831 \\
Recall & 0.920 & 0.931 & 0.835 \\
F-Measure & 0.918 & 0.930 & 0.830 \\
MCC & 0.864 & 0.881 & 0.712 \\
ROC Area & 0.986 & 0.991 & 0.914 \\
PRC Area & 0.975 & 0.985 & 0.857 \\
Class & & high/low/ave & \\
\hline
\end{tabular}




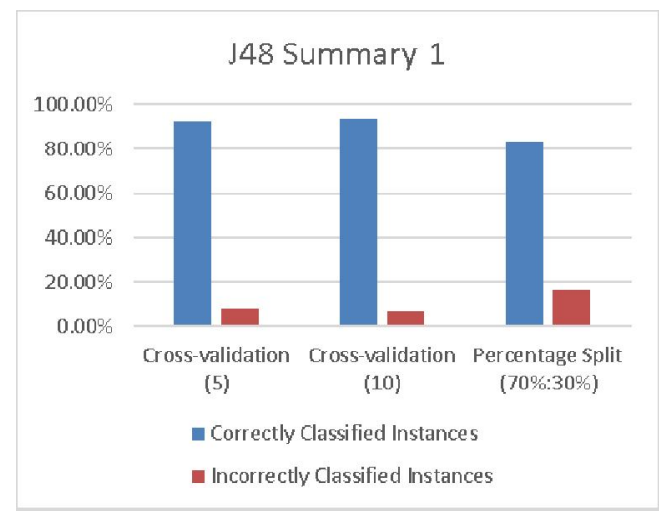

Figure 7: C4.5 Test Options Results Visual Representation

Table 8. RF's results specifics on applied test options

\begin{tabular}{|c|c|c|c|}
\hline Summary & $\begin{array}{c}\text { 5-fold } \\
\text { Cross-vali } \\
\text { dation } \\
\end{array}$ & $\begin{array}{c}\text { 10-fold } \\
\text { Cross-vali } \\
\text { dation } \\
\end{array}$ & $\begin{array}{c}\text { Percentage } \\
\text { Split } \\
(\mathbf{7 0 \% : 3 0 \% )}\end{array}$ \\
\hline $\begin{array}{l}\text { Correctly Classified } \\
\text { Instances }\end{array}$ & $\begin{array}{c}523 \\
(95.438 \%)\end{array}$ & $\begin{array}{c}526 \\
(95.9854 \%)\end{array}$ & $151(92.0732 \%)$ \\
\hline $\begin{array}{l}\text { Incorrectly } \\
\text { Classified Instances }\end{array}$ & $24(4.562 \%)$ & $22(4.0146 \%)$ & $13(7.9268 \%)$ \\
\hline Kappa statistic & 0.9137 & 0.9247 & 0.8544 \\
\hline Mean absolute error & 0.0392 & 0.0313 & 0.0676 \\
\hline $\begin{array}{l}\text { Root mean squared } \\
\text { error }\end{array}$ & 0.1198 & 0.1084 & 0.1814 \\
\hline $\begin{array}{l}\text { Relative absolute } \\
\text { error }\end{array}$ & $11.0018 \%$ & $8.7841 \%$ & $18.8351 \%$ \\
\hline $\begin{array}{l}\text { Root relative } \\
\text { squared error }\end{array}$ & $28.3965 \%$ & $25.3986 \%$ & $42.5118 \%$ \\
\hline $\begin{array}{l}\text { Total Number of } \\
\text { Instances }\end{array}$ & & & 164 \\
\hline $\begin{array}{c}\text { Detailed } \\
\text { Accuracy By } \\
\text { Class (Weighted } \\
\text { Avg.) }\end{array}$ & $\begin{array}{c}\text { 5-fold } \\
\text { Cross-vali } \\
\text { dation }\end{array}$ & $\begin{array}{c}\text { 10-fold } \\
\text { Cross-vali } \\
\text { dation }\end{array}$ & $\begin{array}{c}\text { Percentage } \\
\text { Split } \\
(\mathbf{7 0 \% : 3 0 \% )}\end{array}$ \\
\hline TP Rate & 0.954 & 0.960 & 0.921 \\
\hline FP Rate & 0.032 & 0.020 & 0.059 \\
\hline Precision & 0.954 & 0.960 & 0.926 \\
\hline Recall & 0.954 & 0.960 & 0.921 \\
\hline F-Measure & 0.954 & 0.960 & 0.922 \\
\hline $\mathrm{MCC}$ & 0.928 & 0.941 & 0.86 \\
\hline ROC Area & 0.999 & 0.999 & 0.993 \\
\hline PRC Area & 0.995 & 0.996 & 0.990 \\
\hline Class & \multicolumn{3}{|c|}{ high/low/ave } \\
\hline
\end{tabular}

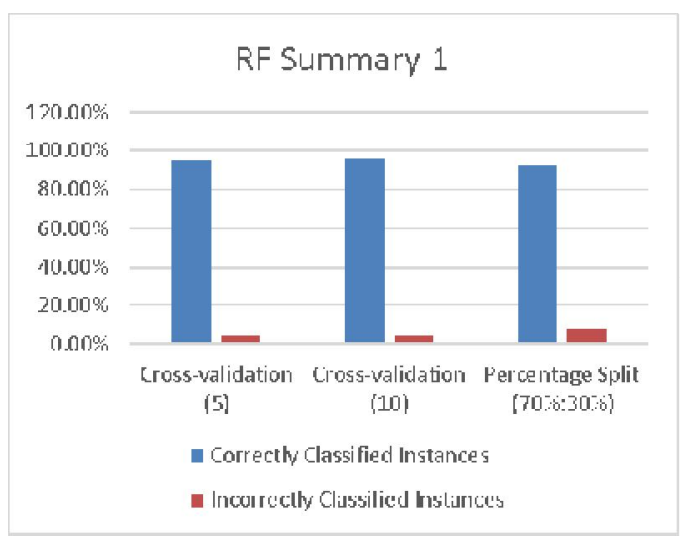

Figure 8: RF Test Options Results Visual Representation

For C4.5 and RF, all Test Options did surpassing results for each classifier. C4.5 to PS of 70\%:30\% showed $83.54 \%$; 5-fold CV showed $91.97 \%$, while 10 -fold CV showed an impressive $93.7 \%$, which revealed that all TO applied to C4.5 gave competitive returns. Last but worth saving, Random Forest. RF to PS of 70\%:30\% showed $92.07 \%$, 5-fold CV showed $95.44 \%$, while 10 -fold CV showed the most with 95.99\% accuracy.

Each classifier had its produced accuracy, which is shown in Figure 9, but all of them proved excellent accuracy on 10-fold Cross-validation. From it, RF to 10 -fold CV made the best.

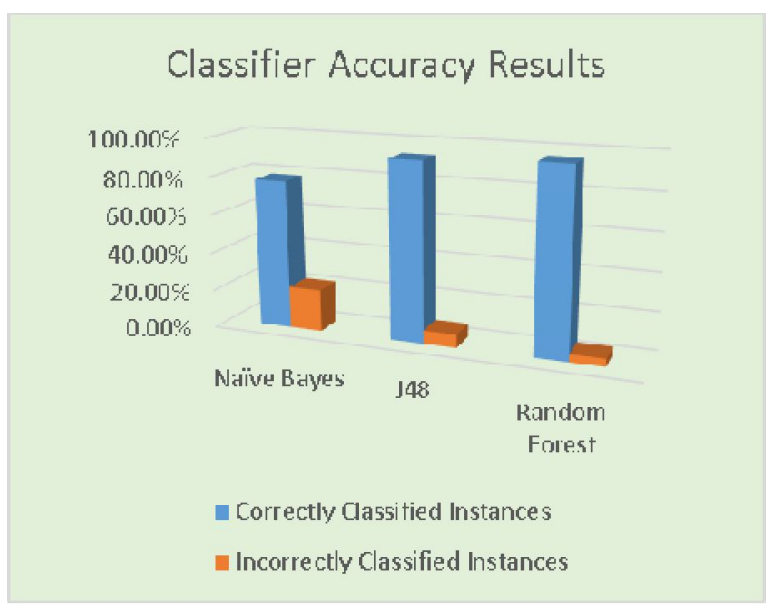

Figure 9: Classifier Accuracy Results from Visualization of $\mathrm{NB}, \mathrm{C} 4.5$, and RF

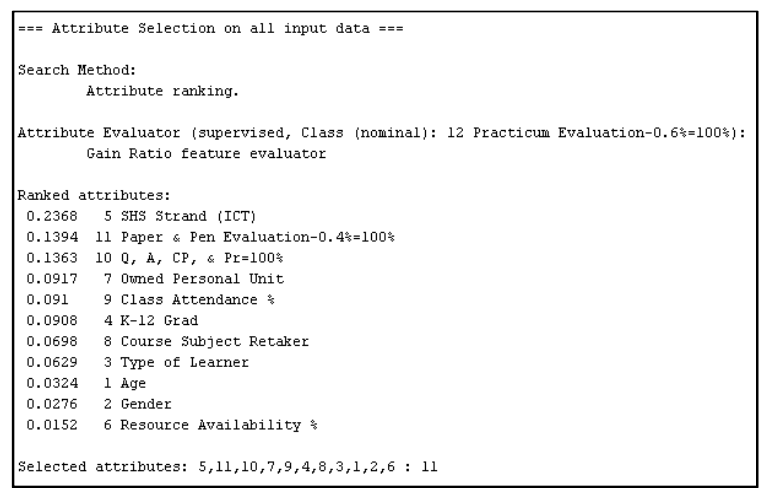

Figure 10: RF as the Classifier with the Most Accuracy Attribute Ranking 
Upon knowing the classifier with the most accuracy, the question about whether which attribute ranked highest in influence. It revealed that with RF's to 10-fold CV, the 5 SHS Strand (ICT) attribute has the most impact in having a higher probability of nailing practical or laboratory examination that is shown in Figure 10.

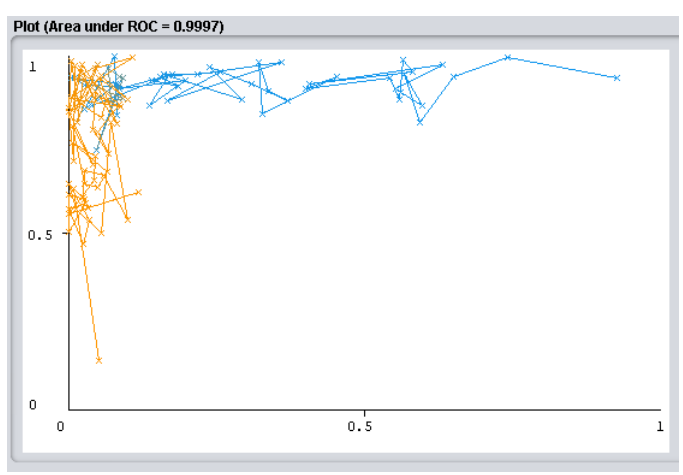

Figure 11: RF as the Classifier with the Most Accuracy Threshold Curve Visualization (Value Class=High)

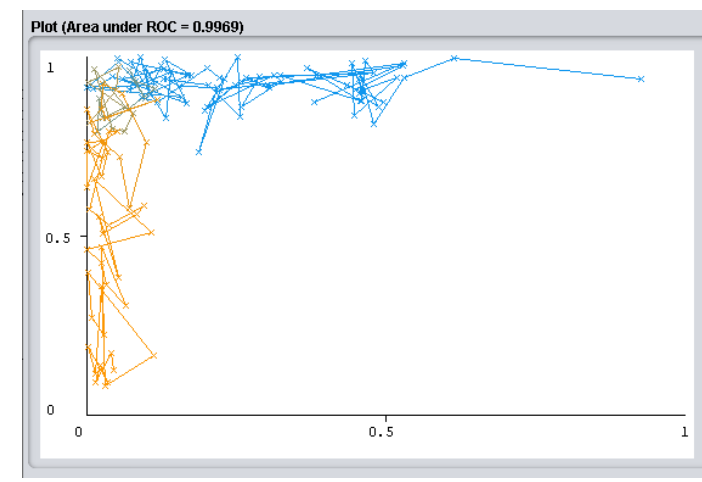

Figure 12: RF as the Classifier with the Most Accuracy Threshold Curve Visualization (Value Class=Ave)

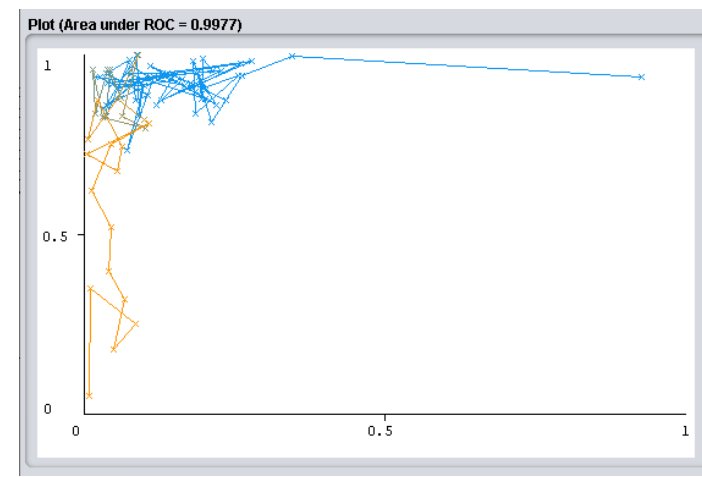

Figure 13: RF as the Classifier with the Most Accuracy Threshold Curve Visualization (Value Class=Low)

Figures 11, 12, and 13 showed that the result values of Plot (Area under Receiving Operating Characteristics [27] or ROC) of all classes (High, Ave, and Low) are close to 1, which means good in terms of measure and performance as per [30].

\section{CONCLUSION}

Throughout the times [27], typically, one of the greatest works in Educational Data Mining is foretelling student academic production [14], [30]. In this study, classification methods were applied in predicting the student record dataset of 549. This study is to anticipate and interpret the likelihood of a student [16] to pass a hands-on examination (laboratory) given with the implementation of OBE teaching-learning methodology.

After data selection, preprocessing, transformation, mining, and evaluation that resulted to an impressive knowledge, the most algorithm that gave a highly satisfactory result is the Random Forest with an accuracy of $95.99 \%$ followed by $\mathrm{C} 4.5$ with an accuracy result of $93.07 \%$ - and Naive Bayes with an accuracy result of $78.1 \%$.

In conclusion, the study met the goal of evaluating student's performance with the noble application of three (3) selected WEKA-based classifiers [14]. Furthermore, additional research applying different popular prediction algorithms and DM methods, or a hybrid, to evaluate the student and assessment performance [27].

\section{REFERENCES}

[1] M. M. Chabeli, "Higher order thinking skills competencies required by outcomes-based education from learners.," Curationis, vol. 29, no. 3, pp. 78-86, 2006, doi: 10.4102/curationis.v29i3.1107.

[2] J. D. Jansen, "Curriculum reform in South Africa: A critical analysis of outcomes-based education," Cambridge J. Educ., vol. 28, no. 3, pp. 321-331, 1998, doi: 10.1080/0305764980280305.

[3] M. Tam, "Outcomes-based approach to quality assessment and curriculum improvement in higher education," Qual. Assur. Educ., vol. 22, no. 2, pp. 158-168, 2014, doi: 10.1108/QAE-09-2011-0059.

[4] G. Brindley, "Outcomes-based assessment in practice: Some examples and emerging insights," Lang. Test., vol. 18, no. 4, pp. 393-407, 2001, doi: $10.1177 / 026553220101800405$.

[5] S. Malan, "The 'new paradigm' of outcomes-based education in perspective," J. Fam. Ecol. Consum. Sci. Tydskrif vir Gesinsekologie en Verbruikerswetenskappe, vol. 28, no. 1, pp. 22-28, 2010, doi: 10.4314/jfecs.v28i1.52788.

[6] D. Z. Deniz and I. Ersan, "An academic decision-support system based on academic performance evaluation for student and program assessment," Int. J. Eng. Educ., vol. 18, no. 2 SPEC., pp. 236-244, 2002.

[7] R. M. Crespo et al., "Aligning assessment with learning outcomes in outcome-based education," in 2010 IEEE Education Engineering Conference, EDUCON 2010, 2010, pp. 1239-1246, doi: 10.1109/EDUCON.2010.5492385.

[8] P. Eric D. Jones, EdD, W. Thomas Southern, PhD, Frederick J. Brigham, "Curriculum-Based Assessment:," Interv. Sch. Clin., vol. 33, no. 4, pp. 239-249, 1998, doi: 10.1177/105345129803300407.

[9] G. Brindley, Outcomes-based assessment and reporting in language learning programmes: A review of the issues, vol. 15, no. 1. 1998. 
[10] R. G. Bagnall, "Performance indicators and outcomes as measures of educational quality: A cautionary critique," Int. J. Lifelong Educ., vol. 13, no. 1 , pp. 19-32, 1994, doi: 10.1080/0260137940130103.

[11] W. A. Firestone, D. Mayrowetz, and J. Fairman, "Performance-based assessment and instructional change: The effects of testing in Maine and Maryland," Educ. Eval. Policy Anal., vol. 20, no. 2, pp. 95-113, 1998, doi: 10.3102/01623737020002095.

[12] C. El Moucary, M. Khair, and W. Zakhem, "Improving Student's Performance Using Data Clustering and Neural Networks in Foreign-Language Based Higher Education," Res. Bull. Jordan ACM, vol. 2, no. 3, pp. 27-34, 2011.

[13] A. Peña-Ayala, "Educational data mining: A survey and a data mining-based analysis of recent works," Expert Syst. Appl., vol. 41, no. 4 PART 1, pp. 1432-1462, 2014, doi: 10.1016/j.eswa.2013.08.042.

[14] A. K. M. Pal and S. Pal, "Analysis and Mining of Educational Data for Predicting the Performance of Students," Int. J. Electron. Commun. Comput. Eng., vol. 4, no. 5, pp. 1560-1565, 2013.

[15] B. Kumar and S. Pal, "Analysis and Mining Educational Data to Analyze Students Performance," Int. J. Adv. Comput. Sci. Appl., vol. 2, no. 6, pp. 63-69, 2011, doi: 10.14569/ijacsa.2011.020609.

[16] P. Kaur, M. Singh, and G. S. Josan, "Classification and Prediction Based Data Mining Algorithms to Predict Slow Learners in Education Sector," Procedia Comput. Sci., vol. 57, pp. 500-508, 2015, doi: 10.1016/j.procs.2015.07.372.

[17] S. K. Bindra, A. Girdhar, and I. S. Bamrah, "Outcome based predictive analysis of automatic question paper using data mining," in Proceedings of the 2nd International Conference on Communication and Electronics Systems, ICCES 2017, 2018, vol. 2018-Janua, no. Icces, pp. 629-634, doi: 10.1109/CESYS.2017.8321154.

[18] H. Raza, M. Faizan, A. Hamza, A. Mushtaq, and N. Akhtar, "Scientific Text Sentiment Analysis using Machine Learning Techniques," Int. J. Adv. Comput. Sci. Appl. Vol. 10, No. 12, 2019, vol. 10, no. 12, pp. 157-165, 2019, doi: 10.14569/IJACSA.2019.0101222.

[19] A. Dhankhar, K. Solanki, A. Rathee, and Ashish, "Predicting Student's Performance by using Classification Methods," J. Adv. Trends Comput. Sci. Eng., vol. 8, no. 4, pp. 1532-1536, 2019, doi: https://doi.org/10.30534/ijatcse/2019/75842019.

[20] C. Romero and S. Ventura, "Educational data mining: A review of the state of the art," IEEE Trans. Syst. Man Cybern. Part C Appl. Rev., vol. 40, no. 6, pp. 601-618, 2010, doi: 10.1109/TSMCC.2010.2053532.

[21] S. K. Yadav and S. Pal, "Data Mining: A Prediction for Performance Improvement of Engineering Students using Classification," World Comput. Sci. Inf. Technol. J., vol. 2, no. 2, pp. 51-56, 2012.

[22] B. Pang, L. Lee, and S. Vaithyanathan, "Thumbs up? Sentiment Classification using Machine Learning Techniques," in Proceedings of the ACL-O2 conference on Empirical methods in natural language processing - Volume 10, 2002, no. July, pp. 79-86, doi: https://doi.org/10.3115/1118693.1118704.

[23] J. Vaidya and C. Clifton, "Privacy preserving Naïve Bayes classifier for vertically partitioned data," in SIAM Proceedings Series, 2004, pp. 522-526, doi: 10.1137/1.9781611972740.59.

[24] C. Troussas, M. Virvou, K. J. Espinosa, K. Llaguno, and J. Caro, "Sentiment analysis of Facebook statuses using Naive Bayes Classifier for language learning," in IISA 2013 - 4th International Conference on Information, Intelligence, Systems and Applications, 2013, pp. 198-205, doi: 10.1109/IISA.2013.6623713.

[25] J. Bhatia, A. Girdhar, and I. Singh, "An automated survey designing tool for indirect assessment in outcome based education using data mining," Proc. 5th IEEE Int. Conf. MOOCs, Innov. Technol. Educ. MITE 2017, pp. 95-100, 2018, doi: 10.1109/MITE.2017.00023.

[26] R. Saxena, "EDM: Performance Evaluation of Decision Tree and Clustering Techniques Using WEKA Platform," Usa Weka, vol. 15, no. 2, 2015.

[27] L. A. Quisumbing, "Predicting Student Performance in a Computer Network Certification Program: using the J48 Algorithm," Indian J. Sci. Technol., vol. 12, no. 24, pp. 1-7, 2019, doi: 10.17485/ijst/2019/v12i24/144747.

[28] J. Torres-Niño, A. Rodríguez-González, R. Colomo-Palacios, E. Jiménez-Domingo, and G. Alor-Hernandez, "Improving accuracy of decision trees using clustering techniques," J. Univers. Comput. Sci., vol. 19, no. 4, pp. 484-501, 2013.

[29] Y. Amit and D. Geman, "Shape Quantization and Recognition with Randomized Trees," Neural Comput., vol. 9, no. 7, pp. 1545-1588, 1997, doi: 10.1162/neco.1997.9.7.1545.

[30] T. Mahboob, S. Irfan, and A. Karamat, "A machine learning approach for student assessment in E-learning using Quinlan's C4.5, Naive Bayes and Random Forest algorithms," in Proceedings of the 2016 19th International Multi-Topic Conference, INMIC 2016, 2017, doi: 10.1109/INMIC.2016.7840094.

[31] T. Winters and T. Payne, "What do students know?," Educ. Leadersh., vol. 60, no. 2, pp. 85-86, 2002, doi: 10.1145/1089786.1089802.

[32] M. C. B. Natividad, B. D. Gerardo, and R. P. Medina, "A Career Track Recommender System for Senior High School Students using Fuzzy Logic," Int. J. Adv. Trends Comput. Sci. Eng., vol. 8, no. 3, pp. 2512-2519, 2019, doi: https://doi.org/10.30534/ijatcse/2019/97852019.

[33] P. Baepler and C. Murdoch, "Academic Analytics and Data Mining in Higher Education," Int. J. Scholarsh. Teach. Learn., vol. 4, no. 2, 2010, doi: 10.20429/ijsotl.2010.040217.

[34] N. Katuk, "Student Behavior and Performance in Unsupervised Online Quizzes: A Case of Computer Organization Course," Int. J. Adv. Trends Comput. Sci. Eng., vol. 8, no. 5, pp. 2307-2314, 2019, doi: https://doi.org/10.30534/ijatcse/2019/69852019. 